\title{
Betreuung von \\ Patientinnen und Patienten am Lebensende
}




\section{Herausgeberin}

Schweizerische Akademie

der Medizinischen Wissenschaften

Petersplatz 13

$\mathrm{CH}-4051$ Basel

$\mathrm{T}+41612699030$

mail@samw.ch

www.samw.ch

\section{Gestaltung}

Howald Fosco, Basel

Druck

Gremper AG, Basel

\section{Auflage}

1. - 5. Auflage 11500

6. Auflage 1500 (April 2014)

Alle medizinisch-ethischen Richtlinien der SAMW sind auf der Website www.samw.ch $\rightarrow$ Ethik verfügbar.

(C) SAMW 2013 
Medizin-ethische Richtlinien

\section{Betreuung von Patientinnen und Patienten am Lebensende}

Vom Senat der SAMW genehmigt am 25. November 2004.

Die deutsche Fassung ist die Stammversion.

Per 1. Januar 2013 erfolgte eine Anpassung an das Erwachsenenschutzrecht. 

II. RICHTLINIEN 6

1. Geltungsbereich 6

2. Recht auf Selbstbestimmung 6

2.1. Urteilsfähiger Patient 6

2.2. Nicht urteilsfähiger Patient 6

3. Behandlung und Betreuung 7

3.1. Palliative Care 7

3.2. Behandlungsverzicht oder -abbruch 8

4. Grenzen des ärztlichen Handelns 8

4.1. Beihilfe zum Suizid 9

4.2. Tötung auf Verlangen 9

III. KOMMENTAR 10

$\begin{array}{ll}\text { ad 1. Geltungsbereich } & 10\end{array}$

$\begin{array}{ll}\text { ad 2.1. Urteilsfähiger Patient } & 10\end{array}$

ad 2.2. Nicht urteilsfähiger Patient 10

ad 3.1. Palliative Care 11

ad 3.2. Behandlungsverzicht oder -abbruch 12

ad 4.1. Beihilfe zum Suizid 12

IV. EMPFEHLUNGEN ZUHANDEN DER ZUSTÄNDIGEN $\begin{array}{ll}\text { GESUNDHEITSBEHÖRDEN } & 13\end{array}$

$\begin{array}{ll}\text { Ressourcen } & 13\end{array}$

$\begin{array}{ll}\text { Aus- und Weiterbildung } & 13\end{array}$

V. ANHANG 16

Hinweise zur Ausarbeitung dieser Richtlinien 16 

Menschen in ihrer letzten Lebensphase sind häufig besonders schutz- und hilfsbedürftig. Sie vergegenwärtigen uns die Endlichkeit der menschlichen Existenz. Entscheidungen am Lebensende stellen grosse Anforderungen vor allem an den Patienten ${ }^{1}$ selbst, aber auch an seine Angehörigen, die Ärzte und das Betreuungsteam. Anliegen dieser Richtlinien ist es, Aufgaben, Möglichkeiten und Grenzen der Betreuung von Patienten am Lebensende aufzuzeigen. Das grundlegende Ziel besteht darin, Leiden zu lindern und die bestmögliche Lebensqualität des Patienten sowie eine Unterstützung der Angehörigen zu gewährleisten.

Im Unterschied zur letzten Fassung der Richtlinien von 1995 wird im Folgenden ausschliesslich auf die Situation sterbender Patienten Bezug genommen. Die Richtlinien zur Behandlung und Betreuung von zerebral schwerst geschädigten Langzeitpatienten wurden entsprechend erstmals separat formuliert. Da sich dennoch gemeinsame Fragen und Probleme ergeben, sei die Bedeutung dieser Richtlinien für die hier im Zentrum stehenden Fragen hervorgehoben. Das Gleiche gilt auch für die Richtlinien zu Grenzfragen der Intensivmedizin und für die Richtlinien zur Behandlung und Betreuung von älteren, pflegebedürftigen Menschen.

Bezüglich der speziellen Problematik der sehr unreifen Frühgeborenen sei auf die Empfehlungen der Schweizerischen Gesellschaft für Neonatologie ${ }^{2}$ verwiesen.

1 Die entsprechenden Texte betreffen immer beide Geschlechter der genannten Personengruppen.

2 Vgl. die Empfehlungen «Perinatale Betreuung an der Grenze der Lebensfähigkeit zwischen 22 und 26 vollendeten Schwangerschaftswochen». Schweiz Ärztezeitung. 2012; 93(4): 97-100. 


\section{Geltungsbereich ${ }^{3}$}

Die Richtlinien betreffen die Betreuung von Patienten am Lebensende. Damit sind Kranke gemeint, bei welchen der Arzt aufgrund klinischer Anzeichen zur Überzeugung gekommen ist, dass ein Prozess begonnen hat, der erfahrungsgemäss innerhalb von Tagen oder einigen Wochen zum Tod führt.

Bei Neugeborenen, Kindern und Jugendlichen am Lebensende gelten die gleichen Grundsätze; insoweit hier besondere Aspekte zu berücksichtigen sind, werden diese in den entsprechenden Abschnitten vermerkt.

\section{Recht auf Selbstbestimmung}

Jeder Patient hat das Recht auf Selbstbestimmung. Die frühzeitige, umfassende und verständliche Aufklärung des Patienten oder seiner Vertreter über die medizinische Situation ist Voraussetzung für die Willensbildung und Entscheidfindung. Dies bedingt eine einfühlsame und offene Kommunikation und die Bereitschaft des Arztes, die Möglichkeiten und Grenzen sowohl der kurativen Behandlung wie auch der Palliative Care zu thematisieren.

\subsection{Urteilsfähiger Patient}

Die Respektierung des Willens des urteilsfähigen Patienten ist zentral für das ärztliche Handeln. Demzufolge ist eine ärztliche Behandlung gegen den erklärten Willen des urteilsfähigen Patienten unzulässig. Dies gilt auch dann, wenn dieser Wille dessen wohlverstandenen Interessen aus der Sicht Aussenstehender zuwiderzulaufen scheint. Auch Minderjährige oder verbeiständete Personen können bezüglich Behandlungseinwilligung urteilsfähig sein.

\subsection{Nicht urteilsfähiger Patient}

Ist es dem Patienten nicht mehr möglich, seinen Willen zu äussern, muss der behandelnde Arzt sicherstellen, dass abgeklärt wird, ob eine Patientenverfügung ${ }^{4}$ vorhanden ist. Liegt eine Patientenverfügung vor, sind die darin enthaltenen Weisungen zu beachten. Hat sich der Patient in Bezug auf die in Frage stehende medizinische Massnahme nicht geäussert, so erstellt das Betreuungsteam unter

3 Mit Aufnahme in die Standesordnung der FMH werden die Richtlinien für FMH-Mitglieder verbindliches Standesrecht.

4 Vgl. auch «Patientenverfügungen». Medizin-ethische Richtlinien und Empfehlungen der SAMW. 
Einbezug der vertretungsberechtigten Person ${ }^{5}$ einen Behandlungsplan. Der Arzt informiert die vertretungsberechtigte Person über alle Umstände, die im Hinblick auf die vorgesehenen medizinischen Massnahmen wesentlich sind. Der Entscheid über den Behandlungsvorschlag wird letztlich von der Vertretungsperson getroffen. Diese muss nach dem mutmasslichen Willen und den Interessen des Patienten entscheiden. Damit lastet eine hohe Verantwortung auf einer emotional stark involvierten Person. Es sollte deshalb oberstes Ziel sein, bezüglich Behandlungsvorschlag einen von allen Seiten getragenen Konsens zu erzielen.

Bei schwierigen Entscheiden kann eine ethische Unterstützung ${ }^{6}$ hilfreich sein.

\section{Behandlung und Betreuung}

\subsection{Palliative Care $^{7}$}

Patienten in der letzten Lebensphase haben ein Anrecht auf Palliative Care. Diese umfasst alle medizinischen und pflegerischen Interventionen sowie die psychische, soziale und seelsorgerliche Unterstützung von Patienten und Angehörigen, welche darauf abzielen, Leiden zu lindern und die bestmögliche Lebensqualität des Patienten zu gewährleisten.

Eine zentrale Aufgabe des Betreuungsteams besteht in einer wirksamen Symptomtherapie. Dazu gehören auch das Eingehen auf Nöte sowie die Verfügbarkeit und die Begleitung für den Patienten und seine Angehörigen. Alle potentiell hilfreichen technischen und personellen Ressourcen (z.B. Fachpersonen für psychische, soziale und seelsorgerliche Begleitung) sollen bei Bedarf zugezogen werden. Palliative Care soll frühzeitig und überall angeboten werden, wo der Patient sich befindet (im Spital oder einer anderen Institution, zu Hause).

Der Arzt ist verpflichtet, Schmerzen und Leiden zu lindern, auch wenn dies in einzelnen Fällen zu einer Beeinflussung (Verkürzung oder Verlängerung) der Lebensdauer führen sollte. Bei therapierefraktären Symptomen kann gelegentlich eine Sedation notwendig werden. Hierbei ist zu beachten, dass nur soweit sediert werden soll, als dies zur Linderung der Symptome nötig ist.

5 Das Gesetz erklärt folgende Personen bei medizinischen Massnahmen als vertretungsberechtigt: In erster Linie Personen, die in einer Patientenverfügung oder in einem Vorsorgeauftrag bezeichnet wurden ( $V$ Vertreter»), in zweiter Linie der Beistand mit einem Vertretungsrecht bei medizinischen Massnahmen, danach Angehörige und weitere Bezugspersonen, die dem Patienten regelmässig persönlich Beistand leisten (Ehegatte bzw. eingetragener Partner, Personen im gleichen Haushalt, Nachkommen, Eltern, Geschwister). Für minderjährige Patienten sind die Inhaber der elterlichen Sorge vertretungsberechtigt.

6 Vgl. "Ethische Unterstützung in der Medizin». Medizin-ethische Empfehlungen der SAMW.

7 Vgl. "Palliative Care». Medizin-ethische Richtlinien und Empfehlungen der SAMW. 
Entscheidungen über Behandlung und Betreuung sollen, wenn immer möglich, vom Betreuungsteam und von den Angehörigen des Patienten mitgetragen werden. Wichtig ist der Einbezug der Angehörigen unter Anerkennung ihrer Doppelrolle als Betreuende und Betreute.

Wünsche nach einer persönlichen Gestaltung der letzten Lebensphase sollen unterstützt werden. Die Betreuung soll auch die Begleitung der Angehörigen, in manchen Fällen über den Tod des Patienten hinaus, umfassen.

Es ist für einen respektvollen Umgang mit dem Verstorbenen zu sorgen; den kulturellen und religiösen Ritualen der Hinterbliebenen soll nach Möglichkeit Raum gewährt werden.

\subsection{Behandlungsverzicht oder -abbruch}

Angesichts des Sterbeprozesses kann der Verzicht auf lebenserhaltende Massnahmen oder deren Abbruch gerechtfertigt oder geboten sein. Bei der Entscheidfindung spielen Kriterien wie Prognose, voraussichtlicher Behandlungserfolg im Sinne der Lebensqualität sowie die Belastung durch die vorgeschlagene Therapie eine Rolle.

Bei Neugeborenen, Säuglingen und Kleinkindern gelten grundsätzlich die gleichen Überlegungen. Erschwerend ist allerdings der Umstand, dass eine Orientierungsmöglichkeit am mutmasslichen Willen oder der Persönlichkeit entfällt. Der Einsatz belastender Massnahmen zur Aufrechterhaltung von Vitalfunktionen muss deshalb vor allem von der Prognose abhängig gemacht werden. Dabei soll die Belastung durch die Therapie in Form von Schmerzen, Unwohlsein und Einschränkung gegen den durch sie voraussichtlich ermöglichten Gewinn an Wohlbefinden, Beziehungsmöglichkeiten und Erlebnisfähigkeit abgewogen werden.

\section{Grenzen des ärztlichen Handelns}

Die Respektierung des Patientenwillens stösst dann an ihre Grenzen, wenn ein Patient Massnahmen verlangt, die unwirksam oder unzweckmässig sind oder die mit der persönlichen Gewissenshaltung des Arztes, mit der ärztlichen Standesordnung oder dem geltenden Recht nicht vereinbar sind. 


\subsection{Beihilfe zum Suizid}

Gemäss Art. 115 des Strafgesetzbuches ist die Beihilfe zum Suizid straflos, wenn sie ohne selbstsüchtige Beweggründe erfolgt. Dies gilt für alle Personen.

Die Rolle des Arztes besteht bei Patienten am Lebensende darin, Symptome zu lindern und den Patienten zu begleiten. Es ist nicht seine Aufgabe, von sich aus Suizidbeihilfe anzubieten, sondern er ist im Gegenteil dazu verpflichtet, allfälligen Suizidwünschen zugrunde liegende Leiden nach Möglichkeit zu lindern.

Trotzdem kann am Lebensende in einer für den Betroffenen unerträglichen Situation der Wunsch nach Suizidbeihilfe entstehen und dauerhaft bestehen bleiben.

In dieser Grenzsituation kann für den Arzt ein schwer lösbarer Konflikt entstehen. Auf der einen Seite ist die Beihilfe zum Suizid nicht Teil der ärztlichen Tätigkeit, weil sie den Zielen der Medizin widerspricht. Auf der anderen Seite ist die Achtung des Patientenwillens grundlegend für die Arzt-Patienten-Beziehung. Diese Dilemmasituation erfordert eine persönliche Gewissensentscheidung des Arztes. Die Entscheidung, im Einzelfall Beihilfe zum Suizid zu leisten, ist als solche zu respektieren. In jedem Fall hat der Arzt das Recht, Suizidbeihilfe abzulehnen. Entschliesst er sich zu einer Beihilfe zum Suizid, trägt er die Verantwortung für die Prüfung der folgenden Voraussetzungen:

- Die Erkrankung des Patienten rechtfertigt die Annahme, dass das Lebensende nahe ist.

- Alternative Möglichkeiten der Hilfestellung wurden erörtert und soweit gewünscht auch eingesetzt.

- Der Patient ist urteilsfähig, sein Wunsch ist wohlerwogen, ohne äusseren Druck entstanden und dauerhaft. Dies wurde von einer unabhängigen Drittperson überprüft, wobei diese nicht zwingend ein Arzt sein muss.

Der letzte Akt der zum Tode führenden Handlung muss in jedem Fall durch den Patienten selbst durchgeführt werden.

\subsection{Tötung auf Verlangen}

Die Tötung eines Patienten ist vom Arzt auch bei ernsthaftem und eindringlichem Verlangen abzulehnen. Tötung auf Verlangen ist nach Art. 114 Strafgesetzbuch strafbar. 


\section{ad 1. Geltungsbereich}

Gemäss dieser Definition sind Patienten am Lebensende zu unterscheiden von Patienten mit unheilbaren, progressiv verlaufenden Krankheiten, insofern sich deren Verlauf über Monate oder Jahre erstrecken kann. Mit den klinischen Anzeichen ist die Gesamtheit der Beobachtungen, zum Beispiel sich verschlechternde Vitalfunktionen, prognostisch ungünstige objektive Befunde und die Beurteilung des Allgemeinzustandes gemeint, die den Beginn des Sterbeprozesses charakterisieren. Es ist allerdings hervorzuheben, dass der Eintritt der Sterbephase nicht selten mit ärztlichen Entscheidungen zum Behandlungsabbruch oder -verzicht im Zusammenhang steht, so dass eine Abgrenzung stets mit gewissen Unschärfen verbunden bleibt.

\section{ad 2.1. Urteilsfähiger Patient}

Folgende Kriterien helfen, die Urteilsfähigkeit gemäss Art. 16 Zivilgesetzbuch festzustellen:

- die Fähigkeit, Information in Bezug auf die zu fällende Entscheidung zu verstehen;

- die Fähigkeit, die Situation und die Konsequenzen, die sich aus alternativen Möglichkeiten ergeben, richtig abzuwägen;

- die Fähigkeit, die erhaltene Information im Kontext eines kohärenten Wertsystems rational zu gewichten;

- die Fähigkeit, die eigene Wahl zu äussern.

Die Urteilsfähigkeit wird im Hinblick auf eine bestimmte Handlung abgeschätzt (und zwar im Zusammenhang mit dem Komplexitätsgrad dieser Handlung); sie muss im Moment des Entscheides vorhanden sein.

\section{ad 2.2. Nicht urteilsfähiger Patient}

Handeln im mutmasslichen Willen des Patienten

Der mutmassliche Wille entspricht dem Willen, den der Patient wahrscheinlich äussern würde, wenn er noch urteilsfähig wäre. Er ergibt sich aus der Bewertung aller feststellbaren Informationen wie Patientenverfügung, Ernennung einer Vertrauensperson, früher gemachten Äusserungen und anderen biographischen Hinweisen. Als Angehörige im Sinne dieser Richtlinien werden die dem Patienten nahe stehenden Personen, insbesondere Ehe- oder Lebenspartner, Kinder oder Eltern und Geschwister, bezeichnet. 
Handeln im wohlverstandenen Interesse des Patienten

Unter «Handeln im wohlverstandenen Interesse (best interest) des Patienten» verstehen wir die Durchführung von medizinisch oder pflegerisch indiziert erscheinenden Massnahmen, denen ein hypothetischer vernünftiger Patient in der entsprechenden Situation voraussichtlich zustimmen würde.

\section{Konfliktsituationen}

Obwohl Angehörige kein Entscheidungsrecht haben, ist im Konfliktfall ein Konsens zu suchen.

\section{ad 3.1. Palliative Care}

\section{Grenzen der Palliativmedizin}

Nicht alles mit Sterben und Tod verbundene Leiden ist vermeidbar. Erkennen und Aushalten der Grenzen sind integrierender Teil der Betreuung des Patienten und seiner Angehörigen. Droht in besonders schwierigen Situationen eine Überforderung des Betreuungsteams, sollte externe professionelle Hilfe in Anspruch genommen werden können.

\section{Beeinflussung der Lebensdauer}

Der «lebensverkürzende Effekt» zentral wirkender Substanzen ist lange Zeit überschätzt worden. Im Allgemeinen sind Schmerzmittel und Sedativa, wenn sie ausschliesslich zur Symptomkontrolle in der letzten Lebenswoche korrekt eingesetzt werden, nicht mit einer Verkürzung der Überlebenszeit assoziiert.

Schmerzmittel und Sedativa können auch missbräuchlich eingesetzt werden, um den Tod herbeizuführen. Es ist aber in aller Regel bereits an der Dosierung resp. Dosissteigerung der Medikamente ein Unterschied zwischen der Schmerz- und Symptomlinderung in palliativer Absicht und der absichtlichen Lebensbeendigung erkennbar.

\section{Weiter- und Fortbildung}

Die Betreuung von Patienten am Lebensende setzt Kenntnisse und Fertigkeiten im Bereich der palliativen Medizin, Pflege und Begleitung voraus. 


\section{ad 3.2. Behandlungsverzicht oder -abbruch}

$\mathrm{Zu}$ den lebenserhaltenden Massnahmen gehören insbesondere die künstliche Wasser- und Nahrungszufuhr, die künstliche Beatmung und die kardiopulmonale Reanimation. Je nach Situation muss auch über Sauerstoffzufuhr, Medikation, Transfusion, Dialyse und operative Eingriffe entschieden werden.

\section{ad 4.1. Beihilfe zum Suizid}

Im Umgang mit dem Wunsch nach Beihilfe zum Suizid in Pflegeheimen sind zudem die Richtlinien und Empfehlungen zur Behandlung und Betreuung von älteren, pflegebedürftigen Menschen ${ }^{8} \mathrm{zu}$ beachten.

Vorgesetzte können ihren Mitabeitern die Beihilfe zum Suizid verbieten, diese oder die Mitwirkung dazu aber nicht von ihnen verlangen.

Der Entscheidungsprozess, der zur Suizidbeihilfe oder zu ihrer Ablehnung führt, muss dokumentiert werden.

Ein Todeseintritt nach Beihilfe zum Suizid muss als ein nicht-natürlicher Todesfall den Untersuchungsbehörden zur Abklärung gemeldet werden. Der Arzt, der Beihilfe zum Suizid geleistet hat, darf nicht selber den Totenschein ausfüllen.

8 Vgl. "Behandlung und Betreuung von älteren pflegebedürftigen Menschen». Medizin-ethische Richtlinien und Empfehlungen der SAMW. 


\section{EMPFEHLUNGEN ZUHANDEN DER ZUSTÄNDIGEN}

GESUNDHEITSBEHÖRDEN

\section{Ressourcen}

Trotz beschränkter Mittel sollten die Verantwortlichen des Gesundheitswesens mit ihrer Politik gewährleisten, dass alle Patienten am Lebensende eine palliative Betreuung im Sinne der Richtlinien erhalten.

Die Institutionen sollten den Auftrag und die Möglichkeit haben, die hierzu notwendigen Voraussetzungen wie Räumlichkeiten, personelle Ressourcen, Begleitung des Betreuungsteams etc. zu schaffen.

\section{Aus- und Weiterbildung}

Die Inhalte der Palliativmedizin und -pflege sollten in die Aus-, Weiter- und Fortbildung aller an der Betreuung von Patienten beteiligten Berufsgruppen integriert werden. 


\section{Hinweise zur Ausarbeitung dieser Richtlinien}

\section{Auftrag}

Am 8. Februar 2002 hat die Zentrale Ethikkommission der SAMW eine Subkommission mit der Ausarbeitung von Richtlinien zur Betreuung von Patientinnen und Patienten am Lebensende beauftragt.

\section{Verantwortliche Subkommission}

Dr. theol. Markus Zimmermann-Acklin, Luzern, Präsident

PD Dr. phil. Jürg Bernhard, Bern

Dr. med. Georg Bosshard, Zürich

Pfrn. Ulrike Büchs, Winterthur

Pflegefachfrau Christine Champion, Moudon

Dr. med. Daniel Grob, Zürich

Prof. Dr. med. Christian Kind, St. Gallen

Dr. med. Hans Neuenschwander, Lugano

Prof. Dr. med. Rudolf Ritz, Basel

lic. iur. Michelle Salathé, Basel (ex officio)

Pflegefachfrau Elisabeth Spichiger, Bern

Dr. med. Philipp Weiss, Basel

Prof. Dr. med. Michel Vallotton, Genf, Präsident ZEK (ex officio)

\section{Beigezogene Experten}

Dr. med. Klaus Bally, Basel

Prof. Dr. med. Verena Briner, Luzern

Prof. Dr. theol. Johannes Fischer, Zürich

Fürsprecher Hanspeter Kuhn, Bern

lic. theol. Settimio Monteverde, Basel

Catherine Panchaud, M.Sc, Puidoux

PD Dr. phil. Klaus Peter Rippe, Zürich

Prof. Dr. iur. et Dr. h.c. Kurt Seelmann, Basel

Prof. Dr. med. Frédéric Stiefel, Lausanne

Prof. Dr. med. Andreas Stuck, Bern

\section{Vernehmlassung}

Am 27. November 2003 hat der Senat der SAMW eine erste Fassung dieser Richtlinien zur Vernehmlassung genehmigt.

\section{Genehmigung}

Die definitive Fassung dieser Richtlinien wurde am 25. November 2004 vom Senat der SAMW genehmigt.

\section{Anpassung}

Die vorliegenden Richtlinien wurden im Jahr 2012 der in der Schweiz ab 1. 1. 2013 gültigen Rechtslage angepasst (Schweizerisches Zivilgesetzbuch; Erwachsenenschutz, Personenrecht und Kindesrecht, Art. 360 ff.; Änderung vom 19. Dezember 2008). 

$\overbrace{0+13}^{\text {CIENT/4 }}$

SAMW

Schweizerische Akademie der Medizinischen

Wissenschaften

\section{ASSM}

Académie Suisse

des Sciences Médicales

ASSM

Accademia Svizzera delle

Scienze Mediche

SAMS

Swiss Academy

of Medical Sciences 